\title{
Application of Kelvin Probe Microscopy for Nitride Heterostructures
}

\author{
R. BożEK* \\ Institute of Experimental Physics, Warsaw University \\ Hoża 69, 00-681 Warsaw, Poland
}

\begin{abstract}
Kelvin probe microscopy is an experimental technique designed to investigate fluctuations of surface potential (work function per electron) related to distribution of electric charge or variations in composition. The paper describes principle and precision of measurements. The results obtained for group-III nitrides semiconductor heterostructures grown on $c$-plane sapphire by metal-organic vapour phase epitaxy are presented. The observations concerns defects: inversion domains for Ga- and N-polar layers, threading dislocations and effects of spontaneous polarization leading to $2 \mathrm{D}$ carrier gas. To achieve insight in the evolution of defects, bevelled and cross-sectioned samples were investigated along with surface of "as grown" layers. Applicability of standard Kelvin probe microscopy method was also extended by investigating dependence of the surface potential on variable wavelength illumination, offering opportunity for spectroscopy of individual defects.
\end{abstract}

PACS numbers: 07.79.- $\mathrm{v}, 61.72 . \mathrm{Hh}, 68.37 .-\mathrm{d}$

\section{Introduction}

Scientific and technological progress in the field of semiconductors is dependent on knowledge on material properties in microscale. High resolution data concerning crystalline structure and composition can be acquired with electron microscopy both scanning (SEM) and transmission (TEM) and accompanying techniques, like energy dispersive X-ray (EDX) spectroscopy. However, until recently it was impossible to investigate directly other properties, like electronic or optical ones. The breakthrough came in 1981 with the invention of scanning tunnelling microscope (STM) [1], which initiated a new branch of microscopy known as scanning probe microscopy (SPM). The idea of SPM was to investigate local properties

*e-mail: rafal.bozek@fuw.edu.pl 
of a sample by observing its interaction with a probe in a form of sharpened tip moved over scanned region. The size of the tip was decisive for lateral resolution. In the case of STM the observed quantity was tunnelling current. In order to enable investigation of forces, probes in the form of a cantilever with a tip at its end was proposed. Observation of the deflection of such cantilever caused by short-range repulsive force was principle of atomic force microscope (AFM) described in 1986 [2]. At present AFM tailored to measure surface topography with the resolution of $1 \mathrm{~nm}$ in the $x-y$ plane and $0.1 \mathrm{~nm}$ in the $z$ direction is the most widely used representative of the SPM family. Soon afterwards application of force microscopy was extended to magnetic (magnetic force microscopy) [3] or electric interactions (electric force microscopy) [4].

Another SPM technique that can be applied for electrical characterization of semiconductors is the Kelvin probe microscopy (KPM) also known as the Kelvin probe force microscopy (KPFM), the Kelvin force microscopy (KFM) or surface scanning potential microscopy (SSPM). The idea of combining the Kelvin probe used for measurements of contact potential difference (CPD) with AFM was for the first time proposed by Nonnenmacher et al. [5], who applied it to measure CPD for metal layers evaporated on a gold substrate. At present KPM is implemented in commercial multimode AFM instruments [6].

\section{Kelvin probe microscopy}

\subsection{Principles of measurements}

A Kelvin probe has usually a form of a capacitor whose plates are made of materials for which the CPD is to be found. Connecting the plates with an external circuit allows flow of electrons from the plate for which the work function is lower to the other one until common Fermi level is obtained. In this way plates become charged and start to attract each other. The CPD can be measured using compensation method by inserting a regulated DC source in the circuit and varying its output until plates become neutral.

KPM uses AFM setup with vibrating cantilever to track interaction within the Kelvin probe. A sample and a conducting AFM cantilever form the required capacitor. To meet the condition of a constant separation between its plates, measurements for each line are performed in two stages. During the first scan profile of the surface is acquired in the standard AFM mode (Fig. 1a). Then the cantilever is returned to the beginning of the line and lifted to the chosen height above the surface (Fig. 1b). During the second passage the cantilever follows the profile at the constant height. It is not excited to oscillate by a piezo, as during the topography scan. Instead external voltage in the form of $V_{\mathrm{DC}}+V_{0} \sin \omega t$ is applied between the tip and the sample. The purpose of the DC component is to compensate CPD. The AC component, with frequency $\omega$ corresponding to the mechanical resonance of the cantilever, is applied to modulate attraction between the cantilever and the sample. 


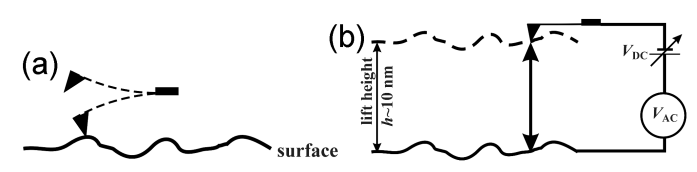

Fig. 1. Principle of operation during (a) topography and (b) surface potential scans.

The force acting between the plates can be found as a derivative of the energy $U$ stored in the capacitor $C$, connected to the source, in respect of separation between plates $z$ :

$$
F=-\frac{\partial U}{\partial z}=-\frac{1}{2} V^{2} \frac{\partial C}{\partial z}
$$

Because in the considered case the work function for both plates has different values, the total potential difference between plates is the sum of the applied voltage and "built in" contact potential difference $V_{\text {cpd }}$ :

$$
V=V_{\text {cpd }}+V_{\mathrm{DC}}+V_{0} \sin \omega_{0} t
$$

Substituting this value to (1) and performing necessary calculations, one can notice that the force between the cantilever and the sample is proportional to the sum of three terms. The first component is independent of frequency, the second depends on resonant frequency $\omega_{0}$, and the third depends on second harmonic $2 \omega_{0}$ :

$$
\begin{aligned}
F \propto & \left\{\left[\left(V_{\mathrm{cpd}}+V_{\mathrm{DC}}\right)^{2}+\frac{1}{2} V_{0}^{2}\right]\right. \\
& \left.+2\left(V_{\mathrm{cpd}}+V_{\mathrm{DC}}\right) V_{0} \sin \omega_{0} t-\frac{1}{2} V_{0}^{2} \cos 2 \omega_{0} t\right\} .
\end{aligned}
$$

The fact that excitation at resonant frequency for which the cantilever is especially sensitive because of high value of its $Q$ factor, is proportional to $\left(V_{\mathrm{cpd}}+V_{\mathrm{DC}}\right)$, offers convenient way to obtain compensation - feedback loop has to adjust $V_{\mathrm{DC}}$ to nullify vibrations of the cantilever at the resonant frequency. In such a case $V_{\text {DC }}$ equals $-V_{\text {cpd }}$.

By definition CPD is a difference between work function for both plates taken per electron. Because the cantilever plays a role of the reference electrode with a fixed value of work function $\Phi_{\text {tip }}$, the measured quantity reflects variations of the work function for the sample $\Phi$ per one electron, i.e. has a dimension of potential. The sign is chosen in such a way that lower potential corresponds to lower work function

$$
V_{\mathrm{cpd}}=-\frac{1}{e}\left(\Phi_{\mathrm{tip}}-\Phi\right)
$$

The work function for semiconductor $\Phi_{\mathrm{sc}}$, which by definition equals the energy required to bring an electron from the Fermi level to the vacuum level, can be expressed by a formula being a sum of three terms

$$
\Phi_{\mathrm{sc}}=\left(E_{\mathrm{c}}-E_{\mathrm{F}}\right)_{\mathrm{bulk}}-e V_{\mathrm{bb}}+\chi .
$$


The terms describe, respectively, relative position of the conduction band and the Fermi level at the depth not influenced by surface, band bending due to charge at surface states and other unscreened charges, and electron affinity being a material property. All these terms point to the sources of contrast in the KPM technique, which can be caused by differences in doping or compensation, composition, distribution and energy levels of defects within depletion layer and treatment of the surface.

The precision of measurements (both in the respect of potential and spatial resolution) is limited by shape and size of a cantilever (length $\approx 225 \mu \mathrm{m}$ and width $\approx 30 \mu \mathrm{m}$ ), which is much greater than characteristic size of investigated fluctuations. The effective force acting on the cantilever is a sum of interactions over its entire surface and only in fraction comes from the sharp tip. For that reason potential found on the basis of minimization of cantilever vibrations gives averaged and thus underestimated value of potential. Influence of shape of the probe on the contrast and fidelity has been considered theoretically and practically by Jacobs et al. [7]. As commercially available probes offer limited choice of shapes, the only way to improve resolution is to minimize tip-sample separation, and thus maximize corresponding tip-sample force.

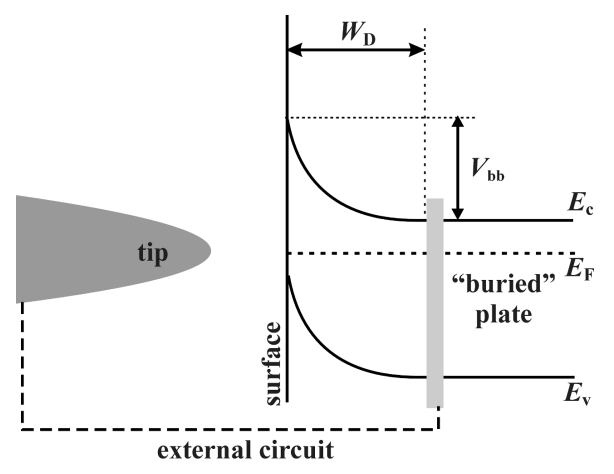

Fig. 2. Configuration of a Kelvin probe at semiconductor surface.

That condition is not easy to be fulfilled for semiconductors. In most cases the Fermi level is pinned at the surface by surface states with energy levels within energy gap, which leads to formation of depletion layer (Fig. 2). Its width $W_{\mathrm{D}}$ increases effective distance between plates of the considered capacitor, significantly worsening resolution. Therefore, while charge distributed within depletion layer affects the surface potential, high resolution can be obtained only if the depletion layer is thin.

\subsection{Surface photovoltage}

The natural extension of the KPM is measurement of light-induced changes in surface potential, called surface photovoltage [8]. Illumination can influence 
surface states reducing band bending and improving resolution. Photoexcited carriers can produce local quasi-Fermi levels in the areas adjacent to the surface of investigated sample, thus revealing fluctuations of potential not visible in dark. Illumination with monochromatic light can selectively change charge on defects e.g. dislocations, allowing to visualize them. In the same way, in case of heterostructures, it is possible to reveal areas with various compositions corresponding to different values of energy gap. It allows identifying layers within heterostructure.

Measurements of surface photovoltage can be done in two different ways. One possibility is to acquire whole image under constant illumination. The other method is photovoltage spectroscopy. It can be accomplished by making the microscope to scan repetitively one, arbitrarily chosen line. If at the same time wavelength of the light is varied, a set of spectra for different points on the chosen line is obtained.

\section{Experiment}

\subsection{Basic properties of nitride epilayers}

The subjects of investigations, whose results are presented in subsequent sections are heterostructures of hexagonal group-III nitrides - gallium nitride GaN and ternary aluminium gallium nitride AlGaN alloys on $c$-plane sapphire. Because the wurtzite structure lacks centre of symmetry, crystals grown with the orientation of the $c$-axis can assume two possible polarities, referred as gallium $(\mathrm{Ga})$ and nitrogen $(\mathrm{N})$ polarity, differing in arrangement of bonds between gallium and nitrogen atoms.

Characteristic feature of hexagonal nitride semiconductors is strong spontaneous polarization in unstrained layers accompanied by piezoelectric polarization appearing under strain $[9,10]$. The vector of spontaneous polarization points along $c$-axis from Ga-face to $\mathrm{N}$-face. Its value is the smallest in the case of GaN and increases with content of aluminium. The consequence of polarization is charge appearing on surfaces or interfaces between layers of different composition.

Ga- and N-polar epilayers differ in many ways. From the point of view of present work relevant are morphology, electrical properties and resistance to chemical etching [11]. Differences in morphology are the consequence of different growth rates for both polarities and presence of inversion domains i.e. inclusions of opposite polarity in Ga- or N-polar layer.

As concerns electrical properties, intentionally undoped GaN grown by metal-organic vapour phase epitaxy (MOVPE) shows usually $n$-type conductivity. However, N-polar layers have distinctly greater concentration of electrons $\left(\approx 10^{17} \mathrm{~cm}^{-3}\right)$ than Ga-polar layers grown under similar conditions. In the latter case concentration shows strong dependence on growth temperature. It is of the order $10^{16} \mathrm{~cm}^{-3}$ for GaN epilayers grown at $1025^{\circ} \mathrm{C}$, but becomes immeasurable (high resistivity GaN), if the growth is conducted at $1100^{\circ} \mathrm{C}$. It is explained 
as result of dependent concentration of gallium vacancies, which being acceptors compensate donors.

Chemical etching in hot aqueous solutions of bases offers simple method of distinguishing between polarities, if the morphology is unclear. N-face of GaN is readily etched in such solutions, while Ga-face remains unchanged [12].

Results obtained due to earlier application of the Kelvin probe microscopy to different epitaxial structures of nitride semiconductors can be found in papers [13-21].

\subsection{Samples}

All investigated samples were grown by low pressure MOVPE using trimethylgallium (TMG), trimethylaluminium (TMA), and ammonia $\left(\mathrm{NH}_{3}\right)$ as precursors. The growth was performed in a generally accepted way starting with low temperature (LT) buffer. After annealing of the buffer $\left(1800 \mathrm{~s}, 1100^{\circ} \mathrm{C}\right)$ under flow of hydrogen and ammonia N-polar layers were obtained by nitridation of sapphire, i.e. annealing of the substrate in $\mathrm{NH}_{3}$ prior to the deposition of LT buffer.

Bevelled samples were prepared by gluing a layer to the appropriate die and polishing first mechanically with diamond pastes and next chemo-mechanically with aqueous potassium hydroxide solution.

\subsection{Inversion domains}

In the case of heteroepitaxial growth of nitrides polarity is not uniquely determined by the substrate. Instead it can be controlled by a treatment of substrate or changing growth conditions of LT buffer [11]. Because those recipes are not perfect, some areas of opposite polarity usually can be observed. Inversion domains (IDs) in nitrides were for the first time reported by Romano et al. [22], who found them with TEM in epilayers grown with various techniques.

So far an attempt to distinguish both polarities with KPM was undertaken by Jones et al. [17], who performed measurements for MBE grown layers, with both polarities obtained simultaneously.

\subsubsection{Ga-face}

Investigations of nitrogen IDs on the Ga-polarity were performed for a series of nominally homogeneous GaN epilayers with various thicknesses. Direct observation was possible for relatively thin layers, approximately $2.2 \mu \mathrm{m}$. Topography of the layer recorded in the AFM mode (Fig. 3a) besides occasional pinholes showed well resolved atomic steps and dislocations pits that could be related to mixed and edge type threading dislocations. The image acquired in the surface potential mode (Fig. 3b) showed more complicated structure, containing numerous depressions in the potential with diameter between 0.5 and $1.0 \mu \mathrm{m}$. These features could not be correlated with dislocations. 

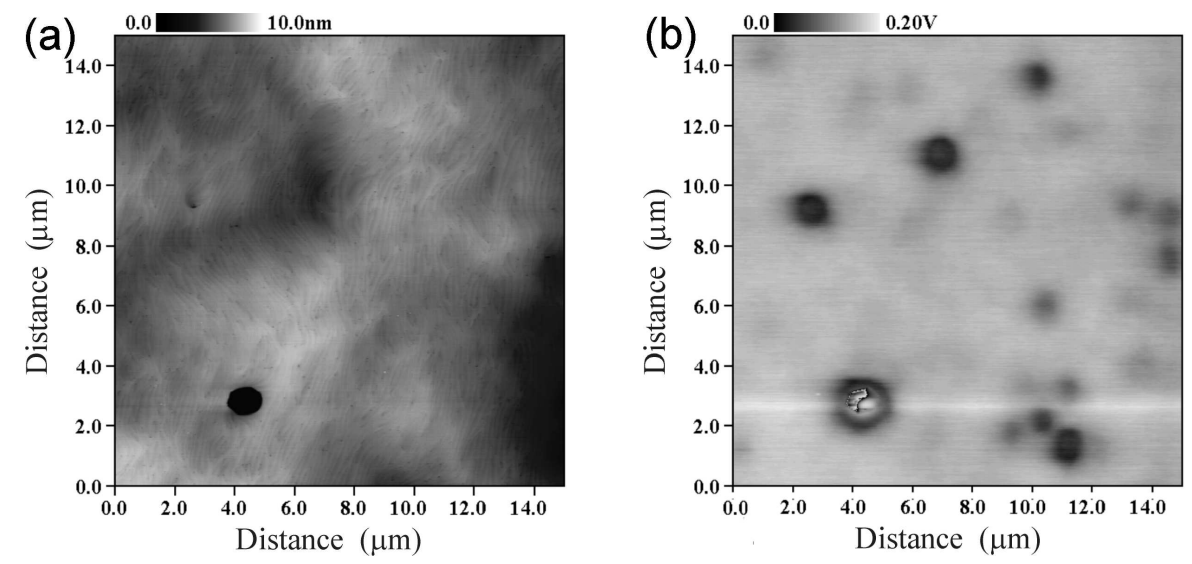

Fig. 3. Images of relatively thin $(2.2 \mu \mathrm{m})$ homogeneous, Ga-polar epilayer of GaN on sapphire acquired in (a) topography and (b) surface potential modes.

Such depressions in potential were not observed for a layer with doubled thickness. In order to find out what is the nature of those fluctuations, further investigations were performed for a sample prepared in the form of a bevel inclined to the $c$-plane at the angle of $\approx 4$ degree, which allowed to observe a layer at different stages of growth. The distance measured along bevel can be connected with the distance by sinus of the angle of inclination.

The acquired topography was featureless besides scratches remaining after polishing. The image registered for a bevel in the KPM mode (Fig. 4) revealed that

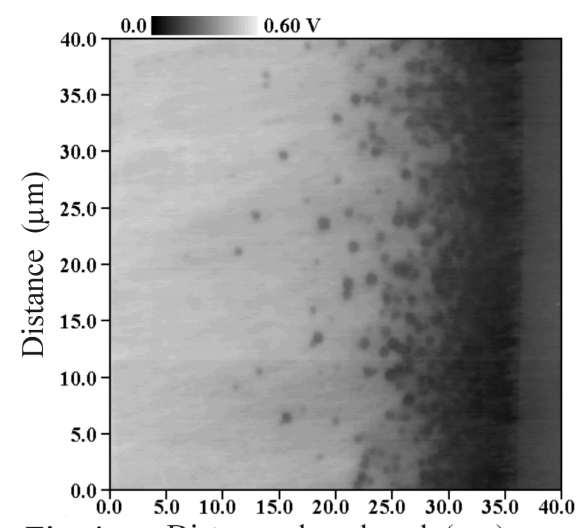

Fig. 4 Distance along bevel $(\mu \mathrm{m})$

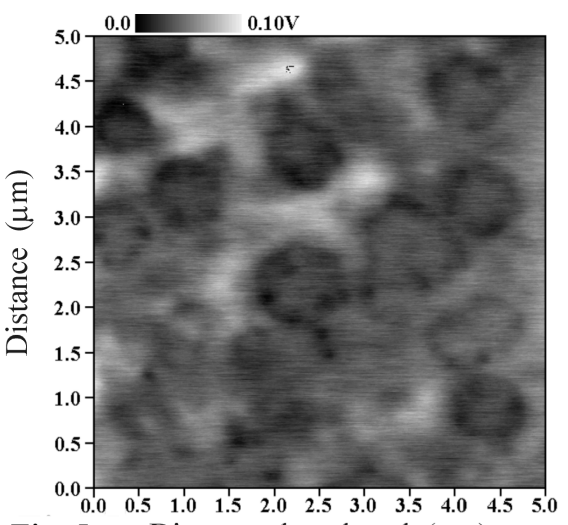

Fig. 5 Distance along bevel $(\mu \mathrm{m})$

Fig. 4. Map of surface potential recorded for a bevelled $4.5 \mu \mathrm{m}$ GaN layer on sapphire (right). The bevel is inclined at 4 degree to the $c$-plane. Distance along bevel (horizontal axis) is related with the distance along $c$-axis by a factor of $\approx 13$.

Fig. 5. Magnified section of the potential image of Fig. 4, taken in the proximity of $\mathrm{GaN} /$ sapphire interface. Structure of hexagonal, ring-like objects related to inversion domains are clearly visible. 
density of depressions in potential was close to $10^{8} \mathrm{~cm}^{-2}$ at the interface with sapphire and decreased with the thickness. They were completely absent in the areas for which distance from the interface (along $c$-axis) exceeded $2.5 \mu \mathrm{m}$. Magnified picture taken in the proximity of LT buffer (Fig. 5) showed that the observed defects were apparently hexagonal and their boundary had lower potential than the inner area. Those findings were confirmed also by measurements of cross-section, which showed rough line of lower potential running at some separation from the LT buffer.

The presented observations can be explained with a simple model connecting depressions with IDs (Fig. 6). If N-polar GaN is overgrown by Ga-polar layer, positive polarization charge appears at the boundary. It attracts free electrons available in N-polar material that tend to screen it. Thus, if the ID boundary is shallowly under the surface - closer than the width of depletion layer, it can be detected with KPM technique as an area of lower potential.

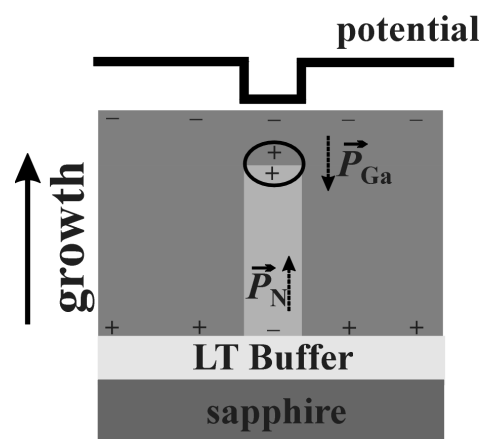

Fig. 6. Model explaining lowering of potential related to overgrown IDs on Ga-polar GaN layer.

Finding that side ID boundaries (IDB) are related to lower potential is consistent with observation identifying them as sources of intense photoluminescence [23]. Also calculations done by Fiorentini [24] showed that IDB can localize both electrons and holes.

\subsection{2. $N$-face}

N-polar GaN epilayers grown by MOVPE are characterized by rough morphology. It is related to Ga-type inversion domains, which grow faster along $c$-axis than surrounding $\mathrm{N}$-face matrix, leading to formation of hexagonal pyramids. Until an ID remains uncovered, the corresponding pyramid preserves sharp peak. In the case when an ID is overgrown, the pyramid stops to grow vertically and transforms into truncated pyramid with flat upper surface. Both types of ID-related pyramids were observed with KPM.

Images recorded for uncovered ID are presented in Fig. 7. Irregularly shaped ID is easily recognizable in topography and surface potential images. In the latter 
it corresponds to peak in the surface potential. It can be easily explained because negative polarization charge at Ga-polar surface increases upward band bending.

Images acquired for a truncated pyramid with an overgrown ID are shown in Fig. 8. This time centre of the pyramid is associated with lowering of potential.
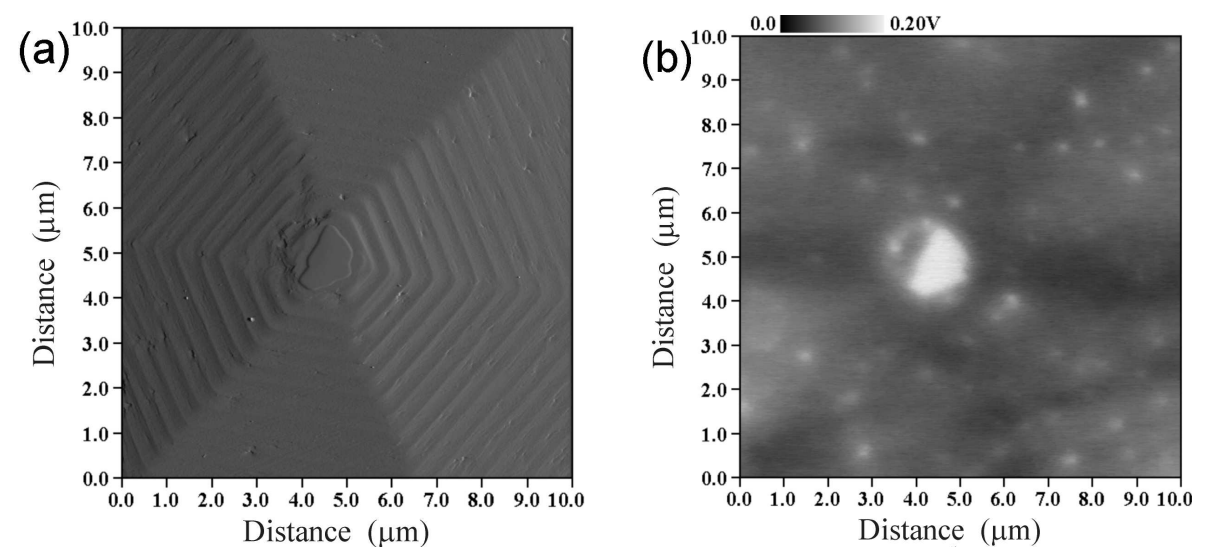

Fig. 7. Images of (a) topography and (b) surface potential for a hexagonal pyramid with uncovered ID on N-polar GaN epilayer. The irregularly shaped ID is visible in the centre. Small bright spots in the potential image correspond to dislocations with large Burgers vector.
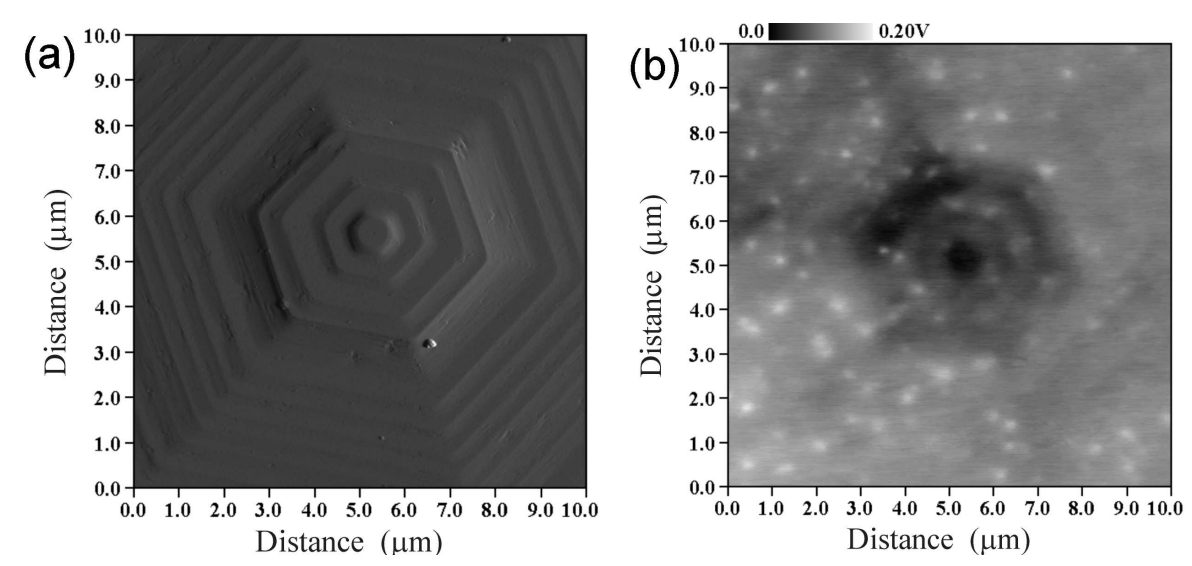

Fig. 8. Images of (a) topography and (b) surface potential for a truncated hexagonal pyramid with an overgrown ID on N-polar GaN epilayer.

Suggested explanation is illustrated with Fig. 9. If N-polar GaN overgrows Ga-polar material, at the boundary negative polarization charge should be found. It tends to be screened by free electrons present in the overgrowing layer. For that 


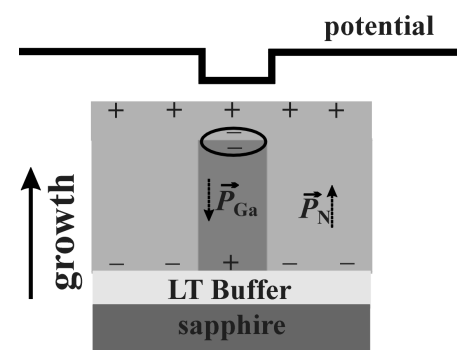

Fig. 9. Model explaining lowering of potential related to overgrown IDs on N-polar GaN layer. Electrons in the covering layer screen the negative polarization charge and shift towards surface.

reason electrons are pushed towards surface, which can be observed as depression in potential.

\subsection{Threading dislocations}

Heteroepitaxial growth of group III-nitride epilayers on substrates with significant lattice mismatch like sapphire, results in high density of threading dislocations, which in the proximity of LT buffer exceeds $10^{10} \mathrm{~cm}^{-2}$ and decreases by 1 or 2 orders of magnitude with thickness. Threading dislocations (TDs) being the most abundant extended defects in nitride heterostructures influence strongly their quality, in part by accommodating electric charges.

Because KPM technique allows for simultaneous recording of topography and potential images, it is possible to distinguish different types of TDs basing on the shape of associated pits and arrangement of atomic steps and in this way correlate them to electric charges. Such attempts undertaken by Koley and Spencer [20] showed correlation of peaks in potential and dislocations pits suggesting neg-

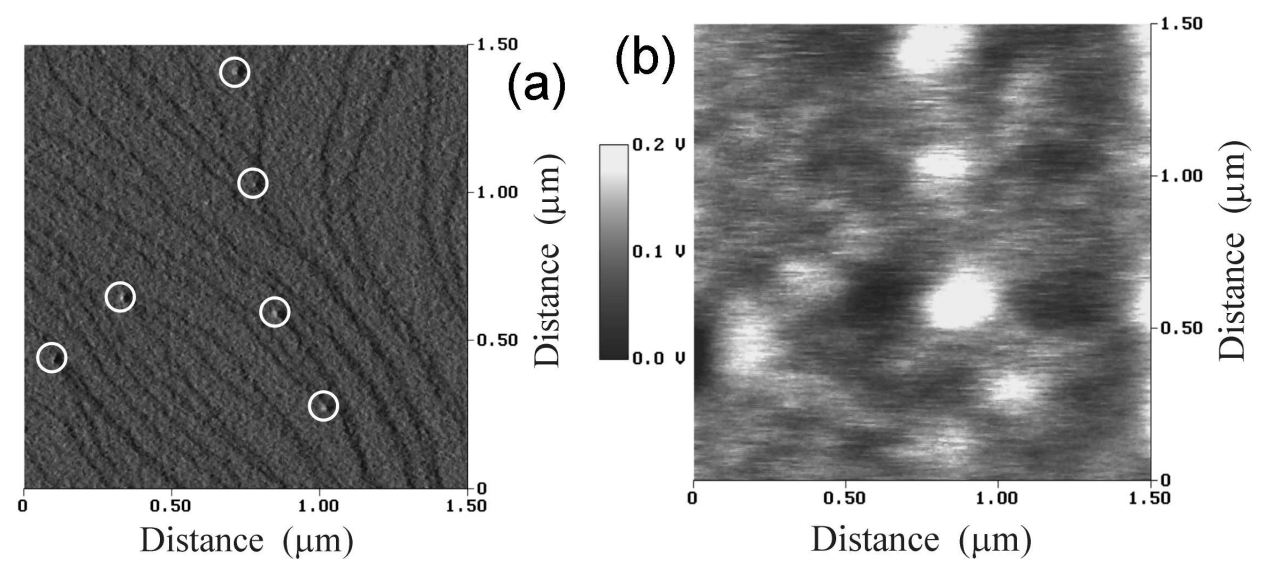

Fig. 10. Images of (a) topography and (b) surface potential for a Ga-polar layer of GaN showing correlation of peaks in potential with dislocation pits of mixed dislocations. Dislocation pits are surrounded with circles. 
ative charge at threading dislocations in $n$-type GaN. Krtschil et al. [19] found dislocations related potential peaks only in $p$-type GaN and failed to observe any contrast for $n$-type layers.

Bożek et al. [21] reported negative charge on mixed type dislocations under illumination with photons of energies close - below and above - energy gap. No peaks were found for pure edge type dislocations. Correlation of mixed dislocations with peaks in potential i.e. negative charge is presented in Fig. 10.

While most measurements point to negative charging of TDs, the problem has not been definitely solved yet. Figure 11 shows topography and surface potential images recorded for $\mathrm{Al}_{0.45} \mathrm{Ga}_{0.35} \mathrm{~N} / \mathrm{Al}_{0.45} \mathrm{Ga}_{0.35} \mathrm{~N}$ :Si grown to obtain a solar
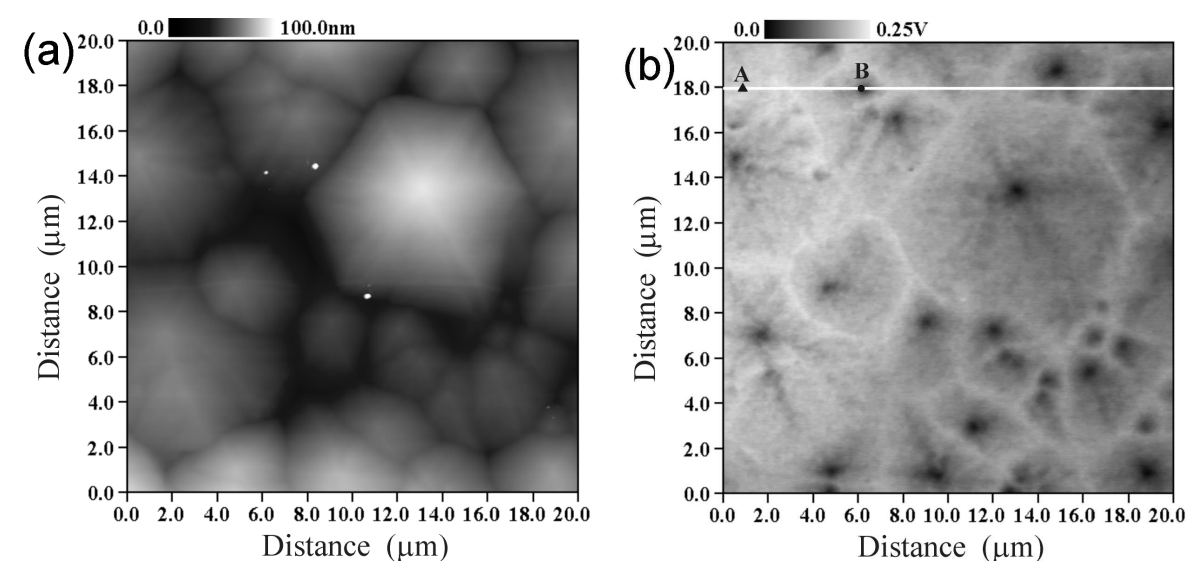

Fig. 11. Images of (a) topography and (b) surface potential for a Ga-polar layer of $\mathrm{Al}_{0.45} \mathrm{Ga}_{0.55} \mathrm{~N}$. Peaks of growth hillocks contain screw dislocations, which correspond to depressions in the potential image. Horizontal line marked in the potential image shows the line, for which spectral dependence of surface potential was measured.

blind detector. Topography of Ga-polar layer shows growth hillocks driven by screw dislocation in the centre of hillocks. The corresponding potential distribution shows lower potential around central dislocation, which suggests positive charge within that region.

\subsection{Photovoltage spectroscopy with KPM}

The same structure has been used to illustrate idea of submicron surface photovoltage spectroscopy performed with KPM as described in Sect. 2.2. The line selected for spectroscopy measurements is marked in Fig. 11b. It passes through a peak of a pyramid i.e. screw dislocation and a point in a valley between neighbouring hillocks.

The spectra obtained at these points are shown in Fig. 12. The difference between the spectra can be attributed to photoionization of dislocation related states. 


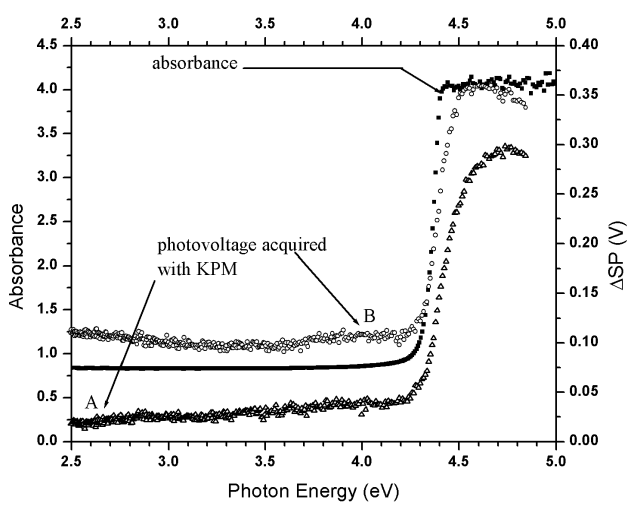

Fig. 12. Spectra recorded for the sample of Fig. 11. Curves consisting of triangles and circles correspond to points respectively marked in Fig. 11. Squares denote absorbance acquired with a spectrophotometer.

For comparison absorbance obtained with a spectrophotometer is presented. It confirms that surface photovoltage can be used to determine composition.

\subsection{Observation of $2 D$-carrier gas}

Another consequence of strong spontaneous and piezoelectric polarization in nitrides is charge induced at interfaces between layers of different composition. The sign of interface charge depends on sequence of layers and their polarity. If polarization charge is positive, it attracts free electrons, which form 2D-electron gas (2DEG). In the case of negative charge and $p$-type conductivity, 2D-hole gas (2DHG) could be formed. Analysis of polarization in

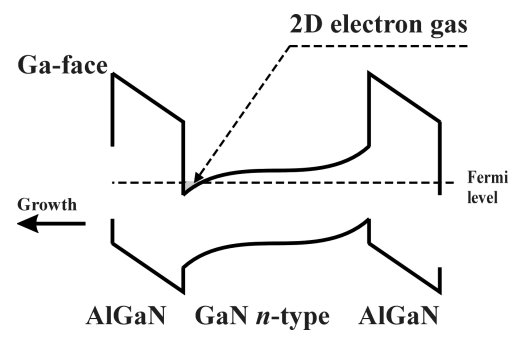

Fig. 13. Scheme of band line-up for AlGaN/GaN/AlGaN structure explaining formation of $2 \mathrm{D}$ electron gas.

$\mathrm{AlGaN} / \mathrm{GaN} / \mathrm{AlGaN} /$ sapphire heterostructure grown with Ga polarity show that AlGaN/GaN interface corresponds to positive and GaN/AlGaN interface to negative polarization charge and respectively (Fig. 13). The presence of 2DEG in such structures has been previously confirmed by Ambacher et al. [25].

Investigations of both interfaces were done for a structure $(1.5 \mu \mathrm{m}$ $\mathrm{AlGaN}) /(0.7 \mu \mathrm{m} \mathrm{GaN}) /(1.5 \mu \mathrm{m} \mathrm{AlGaN})$ grown on sapphire with AlN LT buffer. 


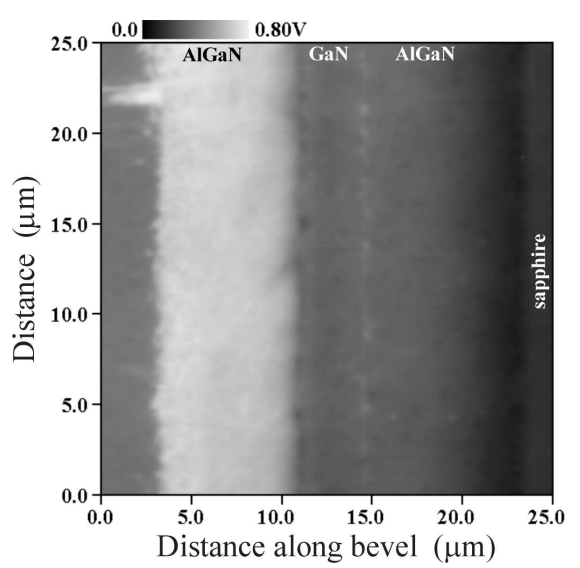

Fig. 14. Map of surface potential recorded for a bevelled AlGaN/GaN/AlGaN heterostructure. The bevel is inclined at 10 degree to the $c$-plane. Distance along bevel (horizontal axis) is related with the distance along $c$-axis by a factor of 5 .

Aluminium content in AlGaN layers was 13\%. The sample used for measurements was prepared as bevel inclined to the $c$-plane at the angle of 10 degree. The map of surface potential, shown in Fig. 14, allows identifying both interfaces. GaN/AlGaN interface is weakly marked as a stripe of slightly increased potential corresponding to negative charge increasing band bending. As there are no free carriers in that area resolution and composition related contrast is poor. Opposite situation is observed at $\mathrm{AlGaN} / \mathrm{GaN}$ interface for which positive polarization attracts free electrons from GaN. The interface is marked stripe of lower potential and strong contrast given by layers with different composition can be seen. It proves that the depleted layer is thin i.e. 2DEG has been formed at that interface.

\section{Conclusions}

The application of Kelvin probe microscopy for investigation of nitrides was described. The method appeared to be particularly useful because many defects produce electric fields because of capturing carriers like in the case of dislocations or because of their relation to polarization charges. Spontaneous and piezoelectric polarization is also responsible for formation of 2D-carrier gas at interfaces, whose property is widely used in nitride based devices. Its observation also was shown with KPM.

At present extended defects in nitrides are most frequently revealed with TEM or chemical etching. However, TEM is not suitable for characterization and applicability of chemical etching is limited by strong dependence of etching properties on crystal orientation. From that point of view KPM technique can become an alternative because it can detect the same electric fields, which are 
responsible for selectivity of etching around defects. The great advantage of the method is a possibility of spectral measurements of individual defects.

\section{References}

[1] G. Binning, H. Rohrer, Ch. Gerber, E. Weibel, Phys. Rev. Lett. 49, 57 (1982).

[2] G. Binning, C.F. Quate, Ch. Gerber, Phys. Rev. Lett. 56, 930 (1986).

[3] Y. Martin, H.K. Wickramasinghe, Appl. Phys. Lett. 50, 1455 (1987).

[4] Y. Martin, D.W. Abraham, H.K. Wickramasinghe, Appl. Phys. Lett. 52, 1103 (1988).

[5] M. Nonnenmacher, M.P. O'Boyle, H.K. Wickramasinghe, Appl. Phys. Lett. 58, 2921 (1991).

[6] Electric Techniques on MultiMode ${ }^{\mathrm{TM}}$ Systems, Support Note 231, Rev. E, Digital Instruments Veeco Metrology Group, 2001.

[7] H.O. Jacobs, P. Leuchtman, O.J. Homan, A. Stemmer, J. Appl. Phys. 84, 1168 (1998).

[8] L. Kronik, Y. Shapira, Surf. Sci. Rep. 37, 1 (1999).

[9] F. Bernardini, V. Fiorentini, D. Vanderbilt, Phys. Rev. B 56, R10024 (1997).

[10] F. Bernardini, V. Fiorentini, Phys. Rev. B 64, 085207 (2001).

[11] M. Sumiya, S. Fuke, MRS Internet J. Nitride Semicond. Res. 9, 1 (2004).

[12] J.L. Weyher, S. Müller, I. Grzegory, S. Porowski, J. Cryst. Growth 182, 17 (1997).

[13] P.M. Bridger, Z.Z. Bandić, E.C. Piquette, T.C. McGill, Appl. Phys. Lett. 74, 3522 (1999).

[14] J.W.P. Hsu, D.V. Lang, S. Richter, R.N. Kleinman, A.M. Sergent, R.J. Molnar, Appl. Phys. Lett. 77, 2873 (2000).

[15] G. Koley, M. Spencer, J. Appl. Phys. 90, 337 (2001).

[16] B.S. Simpkins, D.M. Schaadt, E.T. Yu, R.J. Molnar, J. Appl. Phys. 91, 9924 (2002).

[17] K.M. Jones, P. Visconti, F. Yun, A.A. Baski, H. Morkoç, Appl. Phys. Lett. 78, 2497 (2001).

[18] J.W.P. Hsu, H.M. Ng, A.M. Sergent, S.N.G. Chu, Appl. Phys. Lett. 81, 3579 (2002).

[19] A. Krtschil, A. Dadgar, A. Krost, J. Cryst. Growth 248, 542 (2003).

[20] G. Koley, M.G. Spencer, Appl. Phys. Lett. 78, 2873 (2001).

[21] R. Bozek, K. Pakula, J.M. Baranowski, Phys. Status Solidi C 1, 364 (2004).

[22] L.T. Romano, J.E. Northrup, M.A. O'Keefe, Appl. Phys. Lett. 69, 2394 (1996).

[23] P.J. Schuck, M.D. Mason, R.D. Grober, O. Ambacher, A.P. Lima, C. Miskys, R. Dimitrov, M. Stutzmann, Appl. Phys. Lett. 79, 952 (2001).

[24] V. Fiorentini, Appl. Phys. Lett. 82, 1182 (2003).

[25] O. Ambacher, J. Smart, J.R. Shealy, N.G. Weimann, K. Chu, M. Murphy, W.J. Schaff, L.F. Eastman, R. Dimitrov, L. Wittmer, M. Stutzmann, W. Rieger, J. Hilsenbeck, J. Appl. Phys. 85, 3222 (1999). 